\title{
Left atrial remodelling following transcatheter aortic valve implantation (TAVI) and surgical aortic valve replacement (SAVR)
}

\author{
Tarique A Musa*, Laura E Dobson, Akhlaque Uddin, Timothy A Fairbairn, Ananth Kidambi, David P Ripley, \\ Peter P Swoboda, Bara Erhayiem, Pankaj Garg, Adam K McDiarmid, Sven Plein, John P Greenwood
}

From 18th Annual SCMR Scientific Sessions

Nice, France. 4-7 February 2015

\section{Background}

Left atrial (LA) size is a marker of poor prognosis in a variety of cardiovascular conditions. Severe aortic stenosis results in elevated LV afterload with compensatory hypertrophy and increased filling pressures. This is associated with progressive left atrial enlargement and dysfunction. Intervention for aortic stenosis results in LV reverse remodelling, however the effect of TAVI or SAVR upon LA function remains poorly understood and the two treatments have not been directly compared.

We sought to accurately determine LA size and function using CMR in patients with severe symptomatic aortic stenosis and identify changes following TAVI compared to SAVR.

\section{Methods}

All patients underwent an identical 1.5T CMR protocol (Intera, Phillips Healthcare, Best, The Netherlands). Multi-slice, multi-phase cine imaging was performed using a SSFP pulse sequence in the short axis $(8 \mathrm{~mm}$ thickness, $0 \mathrm{~mm}$ gap, 30 phases, typical field of view (FOV) $340 \mathrm{~mm}$ ) to cover the entire left and right ventricles. All patients were in sinus rhythm at time of CMR imaging; patients with atrial fibrillation were excluded. Offline analysis (CVI42, Circle Cardiovascular Imaging, Calgary, Alberta, Canada) involved measuring the maximum (end systole before mitral valve opening; $L A V_{\max }$ ) and minimum (end diastole right after mitral valve closure; $\mathrm{LAV}_{\text {min }}$ ) LA volumes using the biplane arealength method from the apical 4-and 2-chamber cines.

\footnotetext{
Multidisciplinary Cardiovascular Research Centre \& Leeds Institute for Cardiovascular and Metabolic Medicine, University of Leeds, Leeds, UK
}

Subsequently the total LA emptying fraction was derived as: (LAVmax -LAVmin)*100/LAVmax.

\section{Results}

23 SAVR patients (age $72.7 \pm 7.5$ years, $83 \%$ male, EuroSCORE II $1.40 \pm 1.11 \%$ ) and 23 TAVI patients (age $80.7 \pm 6.9$ years, $57 \%$ male, EuroSCORE II $4.99 \pm 2.97 \%$ ) were studied before and 6 months following valve replacement. The left atria of the TAVI group were significantly more dilated at baseline than those of the SAVR group $(\mathrm{p}=0.039)$ however both groups were comparable at 6 months $(\mathrm{p}=0.227)$. Similarly, the LA emptying fraction of the TAVI group was significantly lower than the SAVR group at baseline $(\mathrm{p}=0.003)$ with comparable function seen at 6 months between the groups $(\mathrm{p}=0.08)$.

\section{Conclusions}

TAVI, but not SAVR, was associated with a significant reduction in LA volume and concomitant improvement in emptying fraction at 6 months. These preliminary findings may reflect worse LA function at baseline in the TAVI group or improved valvular haemodynamics with TAVI compared to SAVR.

Table 1 LA remodelling following TAVI and SAVR

\begin{tabular}{cccc}
\hline TAVI & Baseline & 6 months & $\mathrm{p}$ Value \\
\hline Maximum LA volume (mls/m2) & $63.3 \pm 17.0$ & $52.8 \pm 14.0$ & 0.001 \\
\hline Total LA emptying fraction (\%) & $36.9 \pm 12.6$ & $43.4 \pm 10.4$ & 0.011 \\
\hline SAVR & Baseline & 6 months & $\mathrm{p}$ Value \\
\hline Maximum LA volume (mls/m2) & $53.2 \pm 15.1$ & $48.2 \pm 11.7$ & 0.09 \\
\hline Total LA emptying fraction (\%) & $48.5 \pm 12.8$ & $48.7 \pm 9.1$ & 0.945 \\
\hline
\end{tabular}




\section{Funding}

This study was part-funded by the British Heart Foundation (BHF) (PG/11/126/29321).

Published: 3 February 2015

doi:10.1186/1532-429X-17-S1-P182

Cite this article as: Musa et al:: Left atrial remodelling following

transcatheter aortic valve implantation (TAVI) and surgical aortic valve

replacement (SAVR). Journal of Cardiovascular Magnetic Resonance 2015

17(Suppl 1):P182

Submit your next manuscript to BioMed Central and take full advantage of:

- Convenient online submission

- Thorough peer review

- No space constraints or color figure charges

- Immediate publication on acceptance

- Inclusion in PubMed, CAS, Scopus and Google Scholar

- Research which is freely available for redistribution 